\title{
Comment to Vertical by Giordano CNSY-D-14-00096
}

\author{
William Harkness ${ }^{1}$
}

Received: 27 July 2015 / Accepted: 28 July 2015 / Published online: 19 August 2015

(C) Springer-Verlag Berlin Heidelberg 2015

"Since Mackenzie applied the technique of anatomical hemispherectomy to the surgical treatment of Epilepsy in 1938, there has been considerable interest in hemispheric surgery. The excellent results that can be achieved in the presence of unihemispheric pathology have meant that even when complications to the anatomical approach were described in the 1960 s, surgical ingenuity prevailed and a number of progressively less invasive techniques have since been described. With the increased interest in paediatric epilepsy surgery, these techniques are now being applied to ever younger patients with significant impact not only on seizures but also on development and behaviour. The surgical techniques employed in hemispheric surgery have evolved alongside advances in neuroradiological diagnosis, neurophysiology, neuronavigation and neuroanaesthesia. No single technique has been shown to be clearly superior, each having its own advocates. Nonetheless, in most surgical centres, the detail of the technique employed will depend to some extent on both the pathology for which the procedure is being performed and the skill and experience of the surgical team. In this paper, Giordano and colleagues describe a further variation of hemispherotomy technique which is heavily dependent on neuronavigation and has the objective of lessening intraventricular work and of reducing the chance of hydrocephalus. The authors are to be commended for devising a technique which further 'pushes the envelope', and as a primarily surgi-

This comment refers to the article available at: http://dx.doi.org/10.1007/ s00381-015-2788-1.

William Harkness

William.Harkness@gosh.nhs.uk

1 London, UK cal journal, it is right that Child's Nervous System presents this technique to a wider audience for comment. However, as the paper includes a small number of cases and as the main objective is of a reduced incidence of hydrocephalus, which is already low in all hemispherotomy series, one can only really comment on the technique itself from a surgeon's standpoint. The technique described relies heavily on neuronavigation in direct contrast to every other hemispherotomy technique, which use anatomical landmarks. Hemimegalencephaly and other migrational abnormalities of hemispheric development are common indications for surgery, especially in the younger age group, and for these cases, neuronavigation can present specific challenges which render its accuracy in exact. Also, as the objective of the technique is to remain extra ventricular for the majority of the procedure, this technique would not be suitable for cases where there is a thin cortical mantle, such as MCA infarcts, which represent a significant proportion of surgical candidates. This means that even if clear advantages could be demonstrated in morbidity and outcome, the patient population for this technique is likely to be small. In summary, I believe that all surgeons should continually try and improve the techniques that they use to attempt to reduce morbidity and improve outcomes and constantly explore the literature to bring the latest advances into their practice. In this paper, a novel technique is described, but the reader will have to decide from their own experience whether this represents a significant advance. For those new to the field, I believe that the techniques for hemispherotomy depending upon anatomical landmarks, which are well described in the literature, should act as their starting point and techniques such as this only tried, if deemed appropriate, once considerable experience has been gained." 